\title{
Pyoderma gangrenosum
}
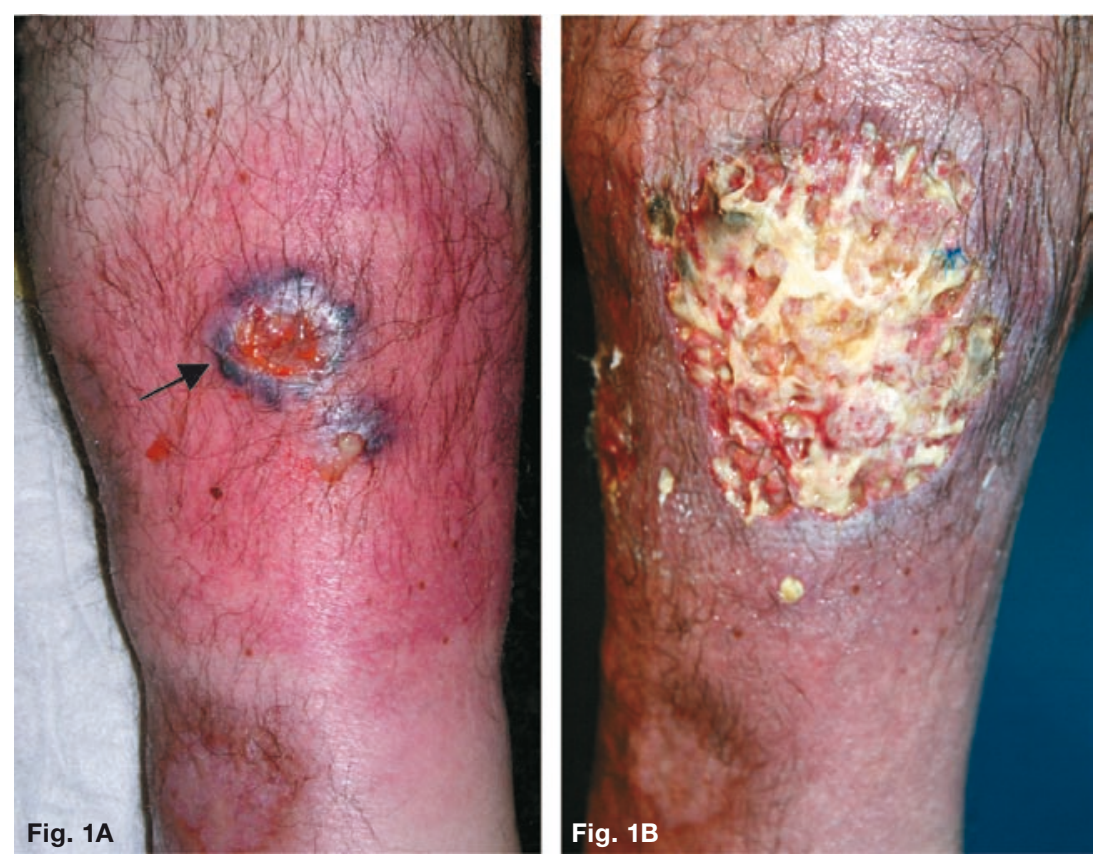

A 42-year-old man presented to our clinic with a two-day history of painful ulcerations on the left lower leg. Physical examination revealed follicular pustulations and tissue necrosis with surrounding erythematous infiltration and edema along with violaceous, undermined ulcer borders (Fig. 1A, arrow). Within 72 hours the patient demonstrated a rapid progression of the skin lesions (Fig. 1B). Colitis ulcerosa had been diagnosed five years ago and was treated with $150 \mathrm{mg}$ azathioprine daily. The medical history enclosed a severe diabetes mellitus and episodes of a schizophrenic disorder, which had been treated with olanzapine $20 \mathrm{mg}$ daily. Dermatohistopathological examinations were consistent with the diagnosis of a pyoderma gangrenosum (PG).

PG is a rare sterile inflammatory neutrophilic dermatosis and is reported to be associated with inflammatory bowel disease (IBD), rheumatic disorders or malignomas [1-2]. Immunosuppression is the most important therapeutic measure in handling PG, along with the treatment of a potentially underlying disease. In our patient we initiated a therapy with topical tacrolimus $0.1 \%$ and systemic glucocorticosteroids up to $100 \mathrm{mg} /$ day with good response after initial enlargement of the ulcer.

Notably, traumatization of the PG (including surgical debridement) has to be avoided, since these measures will cause disease progression in the most cases. Correspondingly, the indication of any other surgical intervention has to be critically considered, since new lesions could be induced at the operation sites.

\section{Norman-Philipp Hoff, Edwin Bölke, Christiane Matuschek, Matthias Peiper,} Norbert J. Neumann, Peter Arne Gerber

Conflict of Interest

The authors declare that there is no conflict of interest.

\section{References}

1. Schuppe HC, Homey B, Assmann T, Martens R, Ruzicka T (1998) Topical tacrolimus for pyoderma gangrenosum. Lancet 351 (9105): 832

2. Ruocco E, Sangiuliano S, Gravina AG, Miranda A, Nicoletti G (2009) Pyoderma gangrenosum: an updated review. J Eur Acad Dermatol Venereol 23 (9): 1008-1017 (Epub 2009 Mar 11)

Correspondence: Peter Arne Gerber, Department of Dermatology, Heinrich-Heine-University Düsseldorf, Moorenstraße 5, 40225 Düsseldorf, Germany, E-mail: PeterArne.Gerber@uni-duesseldorf.de 